\title{
Acute Respiratory Care and Wound Care of Tsunami Patients: What Should the Nurses Do?
}

\author{
Cut Husna
}

Additional information is available at the end of the chapter

http://dx.doi.org/10.5772/51366

\section{Introduction}

The earthquake and tsunami on December 26, 2004 killed more than 270,000 people in the 11 countries, injured 500,000 people, and affected approximately 5 million people in the world. The hardest hit was observed in Aceh province, Indonesia that killed approximately 128,000 people, which is about a quarter of the total population and many people were injured or lost their homes [1]. The 2004 Indian Ocean Tsunami also caused extensive damage to five hospitals in Banda Aceh, 122 hospitals and public health centers in few districts. Health providers and clinical networks beyond the hospitals were left in disarray. Communication and transportation system was disrupted and record of most of the nursing staff was lost. The lack of a medical disaster plan, supply of stockpiles and deployment of personnel were painfully evident in the immediate aftermath of the tsunami [2].

The tsunami has several impacts on physical, psychological, psychosocial, and spiritual aspects of the affected patients. The physical impacts of the affected population such as lung injury, head injury, fracture, wounds, water borne diseases, and insect transmitted diseases. In [3] stated that two-thirds of tsunami patients were reported have combined that injuries to the thorax or fractures. Patients who survived from drowning involved in the aspiration of immersion fluids, marine and soil debris into the respiratory tract and all patients displayed signs of pneumonitis and pneumonia.

The tsunami created dramatic events and the ubiquitous threats of mass destruction. These events have heightened the recognition of the role of nurses in disaster management and response. The health care providers including nurses must response to the tsunami affected patients. The nurses should be able to provide disaster nursing response, communicate with disaster command, and evacuate patients as soon as possible. Unfortunately, most of the nurses are unprepared to respond to the disaster emergency. Many cases indicated inadequate nursing care, medical care, poor communication, chaotic management, and 
meager patient evacuation [4]. For enhancing the capacity to respond to disaster and other public health emergencies, it will require a nursing work force, which is clinically rich in skills and experiences. In addition, nurses should have sufficient skills to respond to tsunami disaster with confidence and authority [5].

The nurses' clinical skills in acute respiratory care involve assessment (airway, breathing, and circulation), intervention (positioning, supplemental oxygenation, fluid management, and antibiotic), and evaluation effectively for airway, breathing, circulation, and disability [6]. Moreover, the nurses' clinical skills in wound care assessment consist of identification characteristic, location, and category of wound. The interventions refer to irrigation, debridement, cleansing, packing, and changing the dressing of wounds [7]. The evaluations are related to evaluate wound condition such as infection, granulation, and signs of wound healing.

In [8], study about wound care for the victims provided by nurses at hospitals of Phang-Nga province, Thailand, found that the nurses had insufficient of knowledge and skills in providing care to the tsunami patients with wounds, particularly while cleaning and suturing the wounds. This results in wounds inflammation and infection due to the penetration of the debris, sand, and mud in the tissues. The nurses also lack of knowledge and technical experiences in dressing the wounds in closed wound with "vacuum assisted closure" (VAC). In reference [9] found that nurses lack of skills in infection prevention and control in mass casualty incident and public education.

Nurses' clinical skills are essential in caring for tsunami patients. Adequate clinical skill helps the nurses in handling the tsunami emergency response effectively. Therefore, the present study assessed the clinical skills for tsunami care and its related factors, and helped in improving clinical skills of nurses in caring for tsunami patients.

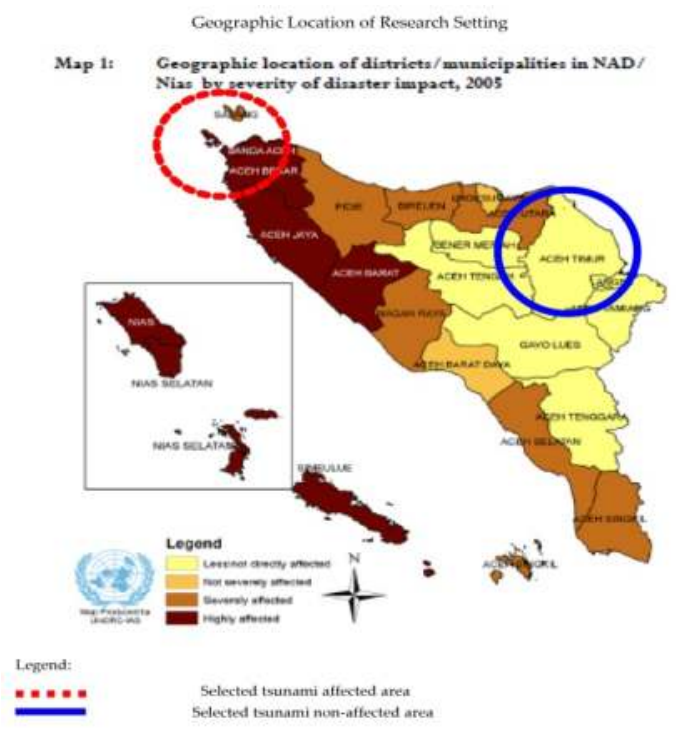

Figure 1. Geographic location of tsunami impact of districts/municipalities in Aceh Province 


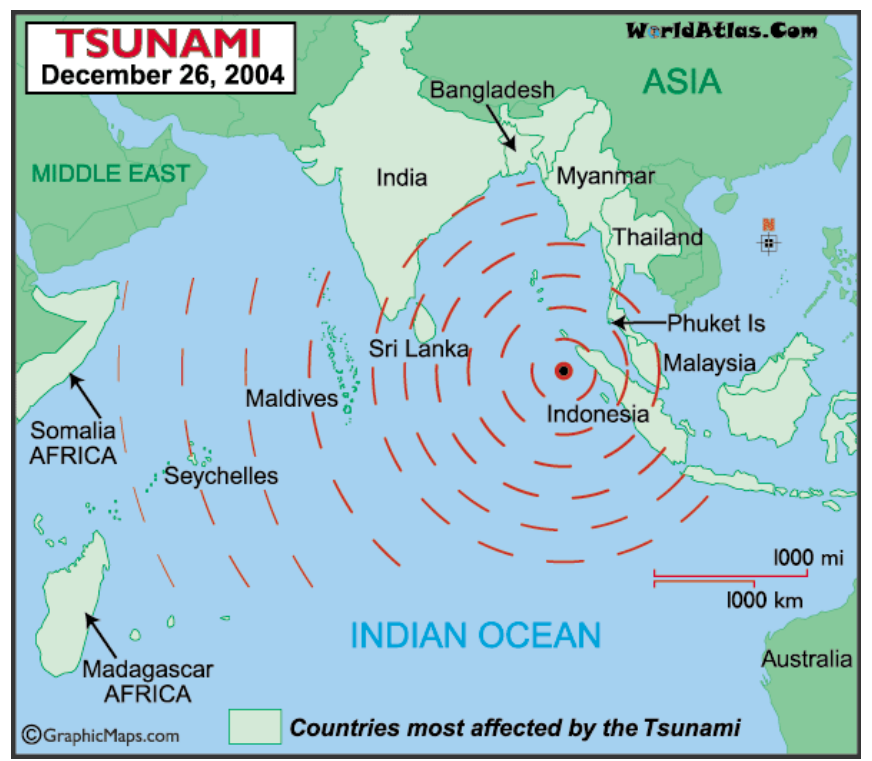

Figure 2. The countries affected by the 2004 Indian Ocean Tsunami

\section{Clinical skills of nurses for tsunami care}

Clinical skills for tsunami care in this study were adopted from its' related concept and two other studies; reference [10] proposed about nurses' competencies in responding to mass casualty incidents/disaster and [11] stated the main problem patients suffering from tsunami trauma.

\subsection{Acute respiratory care}

Aspiration is common problems among tsunami patients due to near drowning. Aspiration pneumonia related with near drowning, is an significant cause of death among tsunami patients. Aspiration of seawater, mud, and marine debris into the respiratory tract provides pathogenic inoculums of pulmonary infection, inducing pneumonitis and pneumonia. Tsunami patients not only aspirate water, but also soil and particulate matters [11].

Respiratory distress syndrome, cardiovascular distress, cardiac arrest, salted water aspiration developed complication associated with near drowning. Near drowning condition caused respiratory and cardiogenic shocks which were common problems encountered in tsunami affected patients [12]. Addressed respiratory tract infections and pneumonias were commonly found several days after the tsunami among the patients who survived from near drowning [13]. Tsunami patients involved in the aspiration of immersion fluids as well as marine and soil debris into the respiratory tract, thus producing intra-pulmonary inoculation of bacteria. In accordance, all patients admitted to hospital displayed radiological and clinical signs of pneumonitis and pneumonia [3]. 
Tsunami lung or tsunami-related aspiration pneumonia is used to describe lung pathology of tsunami patients who have necrotizing, cavitary pneumonia that may create complication with empyema, pneumothorax, and hematogenous spread of infection. Tsunami lung occurs when people being swept by tsunami wave's inhale salt water contaminated with mud and bacteria. The resulting pneumonia like infections normally is treated with antibiotics. However, the 2004-Indian Ocean Tsunami wiped out the medical infrastructure. Therefore, antibiotics to treat the infections in the early stages were not available. Consequently, patients' lung infections festered, entered the bloodstream, and spread to the brain, producing abscesses and neurological problems such as paralysis. The diagnosis of tsunami lung requires chest radiography and computed tomography scan of the brain to confirm abscesses [11]. The diagnosis of tsunami lung requires chest radiography and computed tomography scan of the brain to confirm abscesses [14]. Patients showed high risk of polymicrobial infection of the lungs with various pathogens including Aeromonas spp., Pseudomonas spp., Acinetobacter baumanii, Enterococcus faecium, and Klebsiella pneumonia. The patients were usually affected with a higher frequency of shock, respiratory failure, renal failure, and mortality [11,3].

Many patients after swallowing tsunami water developed aspiration pneumonia. A study in [6] reported that the tsunami patients have developed aspiration pneumonia and showed febrile and shortness of breath. Intervention focused on intravenous fluids, antibiotics, acetaminophen, and supplemental oxygen. Patients with shortness of breath became unresponsive and stopped breathing, were provided with the advanced cardiac life support protocol (cardiopulmonary resuscitation/CPR), intravenous fluids, and several rounds of epinephrine and atropine.

Aspiration pneumonia, inhalation, and injury may develop acute respiratory distress syndrome (ARDS), the most common respiratory problems for tsunami patients. ARDS is a critical and often fatal complication associated with sepsis, trauma, major surgery, and many serious illnesses. The mortality rate has been reached $90 \%$, but that appears to be decreasing with improvements in treatment techniques. Current treatment for ARDS relies on supportive measures to maintain adequate oxygenation until the lungs recover. Conventional therapy includes endotracheal intubation and volume cycled mechanical ventilation [40].

Aspiration caused salted water pneumonia, asphyxia, and acute respiratory distress syndrome. There were totally 2,311 patients attacked by tsunami, approximately $40 \%$ suffered from salted water aspiration, while $2 \%$ developed complication associated with near drowning. Mud, sand, and dirt were seen in the mouth and throat of the victims [12]. In [15] found that there are $62 \%$ patients with acute respiratory infections (62\%) for epidemic-prone disease surveillance and response after the tsunami in Aceh Province, Indonesia.

The nurses have to assess main signs of the tsunami affected patients which includes respiratory distress profound hypoxemia and diffuse bilateral alveolar infiltrate on chest Xray (white lung), breathless, tachycardia, and increase effort of breathing, decrease $\mathrm{PaCO}_{2}$, 
hypoxemia, hypotension, and decrease urine output [17]. The nurses also have to assess for airway, breathing, circulation, and disability in maintaining adequate oxygenation for the patients. Airway is establishing a patent airway by positioning, suctioning, and oxygenation. Breathing related to assess breath sounds and respiratory effort, observe for chest wall trauma or other physical abnormality. Circulation involves monitoring vital signs, especially blood pressure and pulse rate. Disability refers to evaluating the client's level of consciousness (LOC) using ALERT (alert, responsive to voice, responsive to pain, unresponsive) and the GCS, and re-evaluates frequently the client's LOC [17].

In caring for tsunami patients with circulation problems due to acute respiratory distress, the nurses should have sufficient knowledge and skills to assess cardiovascular functions of patients to maintenance circulation, monitoring vital signs and identify signs of shock such as hypovolemic, cardiogenic, and septic shocks. In reference [45] described that shock is the manifestation of the rude unhinging of the machinery of life. Hypovolemic shock associated to cold, clammy skin, reduce peripheral perfusion, tachycardia, oliguria, tachypnea, metabolic acidosis, hypocapnia, and decrease cardiac output. Furthermore, the cardiogenic shock associated to cardiovascular collapse because of the cardiac disease, which is characterized by decrease cardiac output, increase heart rate, tachycardia, vasoconstriction, and increase after load. The septic shock associated with stimulation of the host inflammatory response by an infecting organisms or one of its biological product, the signed with hypovolemic, dissociate, and cardiogenic shock.

The nursing interventions for patients with acute respiratory distress syndrome focused on fluid management involve fluid resuscitation to maintain cardiac output and tissue perfusion. Fluid balance should be considered due to contribution to worsening pulmonary edema. Patients are supported by ventilator support with positive end expiratory pressure (PEEP) to prevent alveolar atelectasis and improve oxygenation, monitor pulmonary artery pressure (PAP) in range $25 / 10 \mathrm{mmHg}$ and pulmonary artery wedge pressures (PAWP) in range 4-12 mmHg [17]. Anti-infective therapies are provided to patients with acute respiratory problems consisting of ampicillin/sulbactam, carbapenemes, fosfomycin, rifampisin, linezolid and glycopeptides [3].

Moreover, altering the patients' position to prone, improve the oxygenation by reducing the ventilation perfusion mismatch and decrease the shunt. Tissue injury of patient can be prevented by changing patients from a supine to a prone position and thus prevents the use of high inspiratory and expiratory pressures [16]. Anti-infective therapies are provided to patients with acute respiratory problems consisting of ampicillin/sulbactam, carbapenemes, fosfomycin, rifampisin, linezolid and glycopeptides [3].

Nurses' clinical skills in acute respiratory care for tsunami care consist of assessing signs and symptoms of near drowning such as dyspnea, cyanosis, and hypothermia, assessing signs and symptoms of pneumonia, removing foreign body from airway, promoting adequate airway by positioning, suctioning, and oxygenation, providing re-warming intervention by using warm blanket or clothing, administering antibiotic therapy for acute respiratory problems, and evaluating patient's condition such as vital signs, breathing, and circulation. 


\subsection{Wound care}

The patients attacked by tsunami showed severe injury, trauma, and wound. The result of the study in [13] showed that wound caused tsunami has specific characteristic: multiple in number, small to medium size, present on the head, face, and extremities, back, buttock and legs. Most of the wounds belong to lower (49\%), and upper extremities (32\%). In reference [3] stated that wounds caused tsunami were significantly contaminated with foreign materials such as seawater, mud, sand, soil, vegetation, stick, and corals inside the cavity. In reference [12] mentioned that several traumas to the parts of the body and extremities were sustained when the wave hit and swept the affected patients. The patterns of injury can be ranged from minor abrasion to large lacerated wound. Fracture, dislocation, and tendon injury were also common problems found in tsunami patients. Debridement was needed in nearly $90 \%$ of the cases and causes of death were near drowning and blunt trauma.

Patients who survived from the tsunami were severely injured, and the incidence of wound infections was high, being at risk of deadly fungal infections and infections caused by waterborne and highly resistant pathogens. The wound displayed the signs such as pus, foul smell, necrosis, gangrene/subcutaneous emphysema, cellulitis, erythema, swelling or infiltration of adjacent tissue, pain, and delayed healing. Many tsunami patients were injured by debris and polluted seawater, which contributed to wound contamination and further tissue damage. Patients mainly suffered from trauma such as wounds, fractures, head injuries, and many needed surgical treatment [18].

A mixture of sea and fresh water, sewage, sand, soil, foreign material causing polymicrobial infection and immediately contaminated wounds. The most common patients are infected by gram-negative pathogen, namely Escherichia coli, Klebsiella, Proteus species, Staphylococci, and Streptococci and infected by bacteria, such as Pseudomonas, Aeromonas, and Shewanella, were probably washed into wounds. Wounds sustained in tsunami disaster are severely contaminated, therefore adequate debridement and delayed suture are recommended for all patients. Anti-infective therapy such as a potent quinolone combined with clindamycin, carbapenemes and glycopeptides were used frequently to control infection $[3,13]$.

Tsunami injuries are categorized into two main groups; soft tissue injury and fracture or dislocation. Soft tissue injuries are classified into minor wounds and major wounds. The minor wound is defined as abrasion, scratch, or small-lacerated wound, which has no need for debridement. The major wounds refer to lacerated wounds with or without skin loss, multiple lacerated wounds, or infected wounds excluded necrotizing fasciitis and open fracture. Fracture and dislocation group included closed or open fracture, single or multiple fractures and dislocation [12]).

For tsunami patients with fractures, the nurses have to assess signs and symptoms of fracture such as local pain, local bleeding, local swelling, deformity or dislocation, numbness, and paralysis. Moreover, the nurses should be able to identify the fracture complications such as bleeding, hemorrhage, shock, and death [19]. The nurses should provide adequate treatment of fracture that involves reduction, immobilization, and 
rehabilitation of the fracture. Reduction aimed to restore alignment and use of internal or external fixation devices to immobilize the fracture. Rehabilitation is particularly important and can prevent complications such as pressure ulcers, deep vein thrombosis, constipation, and urinary stasis. The nursing care for patients with fractures is complex and should include hemodynamic monitoring, pain assessment and management fractures [20].

Many tsunami patients are suffered from trauma and injury in different pain levels. The nurses should have sufficient knowledge and skills to assess tsunami patients for pain levels. In [21] described the numeric pain scale (NPS) which is used to measure pain severity by using whole numbers. The line or bar is marked with whole numbers from 0 to10. Interpretation of pain based on score $0=$ no pain, 1 to $3=$ mild pain, 4 to $7=$ moderate pain, and 8 to $10=$ severe pain. In addition, to assess the pain level effectively, nurses must be able to assess pain severity in diverse condition of patients adequately, understand how to monitor physiologic changes associated with pain and its treatment, be prepared to address the psychosocial experiences accompanying pain and know the consequences of inadequate analgesia [22].

Wound care is an important part of the overall treatment for tsunami patients. Wound care consists of changing the dressing, packing wound, irrigating; debridement process of removing dead tissue, cleansing the wound, or providing a protective environment. The local care, an integral part of treatment of wounds, reduce bacterial burden, exudates, protect the wound from iatrogenic damage, and lead to wound closure. The wound healing care by secondary and tertiary intention includes dressing change, packing, irrigation, and debridement. Types of wound care procedures involve dressing change and reapplication of a covering to the wound. Packing is insertion of material into a wound cavity and covering of the wound with a secondary dressing to prevent invasion of organisms. Irrigation refers to applying fluid under pressure to remove adherent materials and by products of wound metabolism from the surface of the wound. Debridement is removal of necrotic material and slough from the wound [7].

Particularly, to management the wounds caused by tsunami should be focus on surgical removal of devitalized tissue and aggressive debridement. During the interim between initial wound surgery and secondary closure, wound were protected using vacuum-assisted closure (VAC) systems. A major benefit of vacuum-assisted closure system is the reduced need for dressing changes. Furthermore, vacuum-assisted closure therapy draws wounds closed by applying controlled negative pressure while smoothly removing infectious material and interstitial fluids, thus allowing tissue decompression. This system promotes cutaneous perfusion and formation of granulation tissue in the wound. Using this system, definitive wound closure could be achieved within the first week of occurrence of wound [3].

The antibiotic and tetanus prophylaxis are pre-requisite to all patients who have sustained wounds. If the wound is tetanus prone, the patient should also receive tetanus immunoglobulin to prevent tolerance to antibiotics. A tetanus-prone wound is one where the conditions for spore multiplication are favorable. These include deep penetrating and 
puncture wounds where the conditions are likely to be anaerobic, wounds with devitalized tissue, for example, burns, crush injuries, compound fractures, wounds contaminated with dirt or manure, wounds containing foreign bodies, and delayed wound cleaning [23].

Purpose of wound dressing is to provide a condusive environment that will enhance wound healing while offering from trauma. Wound dressings can also help in decreasing or eliminating the pain, reducing the need for dressing changes and providing antibiotic debridement. Choosing the right dressing depend on wound bed characteristics such as wound draining, dry, need moisture, need debridement, and infection [41]. There are some common categories of wound dressings consisting of absorptive dressings, alginate dressings, antimicrobial dressings, biological dressings, collagen dressings, composite dressings, contact layer, hydro gel dressings, impregnated dressings, silicone dressings, transparent film dressings, wound fillers, foam dressings, gauze, non-woven dressings, and hydrocolloid dressings [42].

In caring for tsunami patients with mild and severe injuries, wound dressings may protect the wound from any contaminating agent and microorganism. In reference [42] stated that the gauze may inhibit bacterial penetration and may reduce infection in partial and full thickness of wounds. The gauze, which is easily available and relatively inexpensive, is used widely in wound care. A conservative treatment absorbs minimal exudates and should be placed into the wound in a moist state and removed while still moist. To maintain a moist wound bed, gauze should be moistened with normal saline or a wound gel, but not be so wet that the moisture comes through the cover dressing. The gauze should remain moist while in the wound, so it usually needs to be changed every six hours. When used properly, gauze dressings are conservative and safe on stage II (the topmost layers of skin is severed), III (the fat layer and subcutaneous is severed), and IV (the bone and muscle is severed with death tissue and drainage). The enzymatic debridement is accomplished by coating the wound bed with topical enzymes that digest necrotic tissue.

In reference [43] the nursing management of a client with acute inflammation includes minimizing complications of the edema with inflammation, reducing the inflammatory response, and monitoring systemic response.

\subsubsection{Controlling the effect of edema}

Rest, ice, compression, and elevation (RICE) system can be used to reduce the effect of edema. Ice is used to control the inflammatory response in extremities, particularly when edema and pain are present. Analgesic may be required for pain control.

\subsubsection{Reduce inflammation}

Reduce inflammatory agent may be prescribed to stabilize the mast cell and reduce edema in the affected area, for example non-steroid anti-inflammatory drugs (NSAID). Foreign bodies may be removed to reduce the cause of the inflammation. 


\subsubsection{Monitor system responses}

Temperature is monitored and fever is treated with antipyretics. Client's temperature is monitored closely to prevent harm. The client with a fever may also experience malaise, nausea, anorexia, weight loss, tachypnea, and tachycardia. The diet of the client suffering from inflammation should be high in vitamin C, protein, calories, and fluids.

The most of cleansing agents and dressings that were used for patients with trauma on wounds care were normal saline, providone iodine, hydrogen peroxide, and using contact layer gauze, telfa, and enzymatic debridement. Tsunamis injury causes severe acute pain [7]. In caring for patients with acute pain, the nurses should have sufficient knowledge and skills regarding pain management with pharmacological and non-pharmacological approaches. The pharmacological approach focused on analgesia therapy. For curing pain pharmacologic treatments were used such as opioids, sedatives, non steroidal anti inflammatory drugs (NSAIDs) and local treatment included lidocaine injection and topical eutectic mixture of local anesthetic (EMLA) cream [7].

Furthermore, non-pharmacological treatment focused on physical and psychological approaches. In reference [44] stated the physical therapies and other complementary techniques consisted of massage, reflex zone therapy, acupuncture, shiatsu, therapeutic touch and transcutaneous electric nerve stimulation (TENS). Finally, the psychological therapy includes pre-operative information giving, cognitive methods, distraction, music, humor, hypnosis, relaxation training, guided imagery, and biofeedback. In [7] studied pain management for non-pharmacotherapy showed that using humor (18.9\%), distraction (15.5\%), and deep breathing (12.1\%) were effective in curing pain.

Based on the literature review, nurses' clinical skills in wound care for tsunami care consist of assessing signs and symptoms of contaminated tsunami wound with sand, sewage, and mud, assessing characteristics of tsunami injuries (soft tissue injuries and fractures), assessing bone deformities and bleeding for patients with fractures, assessing pain intensity, cleansing tsunami wound by using normal saline, preparing patients for debridement, administering antibiotic and tetanus prophylaxis, and evaluating signs and symptoms of wound healing such as granulation growth.

\section{Problem statements}

- What is the nurses' clinical skill level of acute respiratory care for tsunami patients among nurses in Banda Aceh, Indonesia?

- What is the nurses' clinical skill level of wound care for tsunami patients among nurses in Banda Aceh, Indonesia?

\subsection{Application area}

The study was focused on disaster nursing in acute response phase on the 2004 Indian Ocean Tsunami in caring patients in the hospital setting. 


\subsection{Research design}

This study was a descriptive explorative study. The aim of this study were to; (1) identify the nurses' clinical skill level of acute respiratory care for tsunami patients among nurses in Banda Aceh, Indonesia, and (2) identify the nurses' clinical skill level of wound care for tsunami patients among nurses in Banda Aceh, Indonesia.

\subsection{Population and setting}

The target population of the study was the nurses who worked in acute care, and emergency and critical care setting from a general hospital in Banda Aceh, Indonesia. The acute care setting consisted of medical ward, surgical ward, and neurological ward. The emergency and critical care setting consisted of emergency department (ED), intensive care unit (ICU), and operating room (OR). Three of these settings were directly involved in response phase for tsunami care patients on December 26, 2004. The total number of the nurses was 195 . The nurses who met the following inclusion criteria were recruited:

- Had been working as permanent and contracted employees of the hospital.

- Had working experience as a nurse at least a year.

\subsection{Sample and sampling}

\section{Sample Size}

The number of respondents in this study was estimated by using power analysis. The sample size was determined at the level of significance $(\alpha)$ of .05 , the power of test $(1-\beta)$ of .80 and the effect size (@) of .30 , which is categorized as medium effect size and is used commonly in nursing research. The sample size of 88 was needed [24]. To avoid low response rate of the sample in the study, the sample size was added by $10 \%$, therefore, the total samples of 97 were recruited.

\section{Sampling Technique}

The samples were recruited using systematic random sampling with odd number of each ward. They were randomly selected from each ward as shown in table 2 . The reasons to perform this technique because it was identified in the sampling frame, the desired sample size, and the size of the population.

\subsection{Instrumentation}

The instruments used in this study were developed by the researcher and colleagues. They have three main parts, 1) the Demographic Data Questionnaire [DDQ], 2) the Nurses' Clinical Experience Questionnaire [NCEQ], 3) the Tsunami Care Questionnaire [TCQ]. 


\subsection{Demographic Data Questionnaire (DDQ)}

The DDQ was used to gather the following data: age, gender, marital status, religion, educational background, training and education, working experience as a nurse, and attending hospital disaster drill. The questionnaire was developed by the researcher.

\section{Training and Education}

The variable training and education was measured by asking respondents to indicate type of trainings and number of times on the particular type of training. Type and number of times were multiplied then summed for all types, producing a training experience index. The higher index score reflects the higher attendance in emergency training and education program.

\section{Attending Hospital Disaster Drill}

Hospital disaster drill was measured by using dichotomous choice questionnaire. The higher score reflects the higher attendance in hospital disaster drill.

- $\quad$ Nurses' Clinical Experience Questionnaire (NCEQ)

Clinical experience of nurses was measured by using Nurses' Clinical Experience Questionnaire (NCEQ). The questionnaire consisted of 10 items which was measured by using a 4-point Likert-like scale, and was scored from 0 to 3 , which $0=$ never, $1=$ sometimes, 2 = often, 3 = always. The higher score reflects the higher experience to perform nursing activities in caring for tsunami patients.

- $\quad$ Tsunami Care Questionnaire (TCQ)

The Tsunami Care Questionnaire was used to measure perceived clinical skills of nurses for tsunami care. The instrument is composed of 15 items with two subscales: acute respiratory care $(1,2,3,4,5,6)$ and wound care $(7,8,9,10,11,12,13,14,15)$.

The respondents were asked to rate each item using a 5-point Likert-like scale, ranging from 1 to 5 where 1 = very poor, 2 = poor, $3=$ fair, 4 = good, and $5=$ excellent. The total score and subscales score are the summed score of all items and items belong to those subscales. They were then averaged for comparable interpretations and categorized into three levels: low $=$ $1.0-2.3$, moderate $=2.4-3.7$, and high $=3.8-5.0$. The higher score reflects the higher clinical skills for tsunami care perceived by nurses.

\subsection{The reliability of the instruments}

Nurses' Clinical Experience Questionnaire (NCEQ) and Tsunami Care Questionnaire (TCQ) were tested for internal consistency by using Cronbach's alpha coefficients yielding the values of .93 , and .98 , respectively. The reliability tests were performed with 20 respondents who had similar criteria to the study respondents. Cronbach's alpha coefficients of the instruments were used with the expected value of at least .70, which were accepted for a new instrument [24]. 


\subsection{Ethical consideration}

The respondents who agreed to participate voluntarily in this study were informed about their right to withdraw in this study at any time for any reasons without negative consequences. The researcher explained the purposes of the study, expectations from the respondents' participation and potential harms in this study such as the feelings of the traumatic recall, sadness, depression, and despair in completing the questionnaires. In this study, no respondents experienced emotional or psychological problems during completing the questionnaires. The researcher explained them how to complete the questionnaires. The researcher maintained anonymity of the respondents by using code and all of the information was kept confidential.

\subsection{Results}

The results of the study will be showed as follow:

- Demographic Characteristics and Experience

The respondents consisted of 97 nurses who worked in the hospital. Most of the respondents were more than 30 years old $(56.7 \%)$ with a mean age of $31.9(\mathrm{SD}=6.6)$. The majority of the respondents were female $(71.1 \%)$, around three-fourth of them were married $(75.3 \%)$, were educated at diploma level $(78.4 \%)$, and all of them were Islam $(100 \%)$. With regard to attending emergency training and education of the respondents had six index scores $(37.1 \%)$ with the mean score of $4.3(\mathrm{SD}=1.9)$. Regarding working experience as a nurse of the respondents, more half of them had experience more than 5 years $(53.6 \%)$ with a mean score of $8.7(S D=7.5)$. Approximately 80.4 percent of the respondents had experience in caring for tsunami patients and 40.2 percent had experience in caring for tsunami patients for 3-4 months with a mean score of 3.0 (SD = 1.9). Obviously, 42.3 percent of the respondents had attended hospital disaster drill with the mean score of $0.4(\mathrm{SD}=0.5)$.

- $\quad$ Level of Perceived Clinical Skills for Tsunami Care

Overall, perceived clinical skills for tsunami care were at a moderate level with the total mean score of $3.52(\mathrm{SD}=0.86)$. The highest mean score was on acute respiratory care $(\mathrm{M}$ $=3.57, \mathrm{SD}=0.86)$ and followed by wound care $(\mathrm{M}=3.50, \mathrm{SD}=0.96)$ (Figure 3 ).

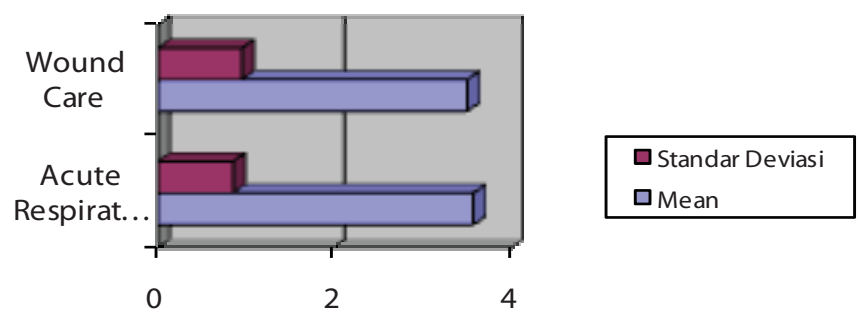

Figure 3. Perceived Clinical Skills 


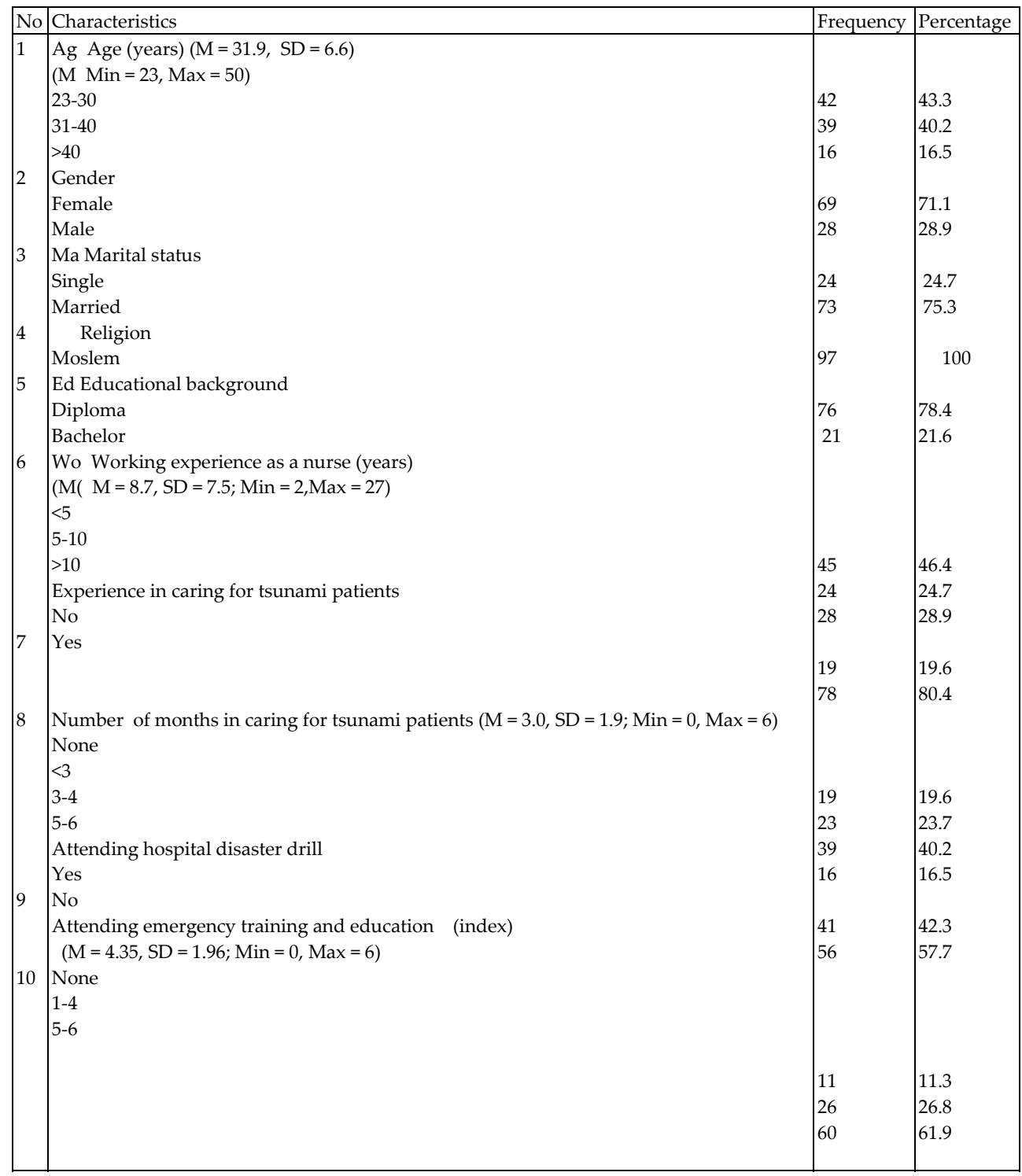

Table 1. The Frequency and the Percentage of Demographic Characteristics and Experience $(\mathrm{N}=97)$

- $\quad$ Perceived Acute Respiratory Care Skills

There was one item of perceived acute respiratory care skills, which had the mean scores at a high level, and the other six items were at a moderate level. The top one items with the highest mean scores was "Administering antibiotic therapy for acute respiratory problems" $(\mathrm{M}=3.82, \mathrm{SD}=1.19)$. The two items with the lowest mean scores were 
"Assessing signs and symptoms of near drowning such as dyspnea, cyanosis, and hypothermia" $(\mathrm{M}=3.33, \mathrm{SD}=0.74)$ and "Providing re-warming intervention by using warm blanket or clothing" $(\mathrm{M}=3.32, \mathrm{SD}=0.96)$, which were at a moderate level (Figure 4).

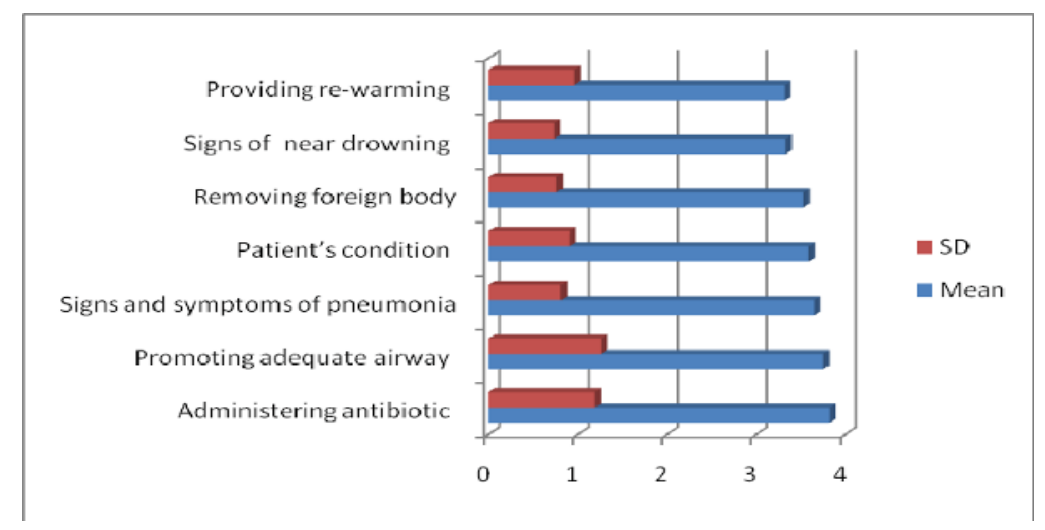

Figure 4. The Levels of Perceived Acute Respiratory Care Skills

- $\quad$ Perceived Wound Care Skills

All items of perceived wound care skills had the mean scores at a moderate level. The one item with the highest mean score was "Cleansing tsunami wound by using normal saline" $(\mathrm{M}=3.67, \mathrm{SD}=1.09)$. The two items which had an equal mean scores included "Assessing signs and symptoms of contaminated tsunami wound with sand, sewage, and mud" $(\mathrm{M}=3.54, \mathrm{SD}=1.25)$, and "Administering antibiotic and tetanus prophylaxis" $(\mathrm{M}=3.54, \mathrm{SD}=1.23)$. The item with the lowest mean score was "Assessing bone deformities and bleeding for patients with fractures" $(\mathrm{M}=3.40, \mathrm{SD}=1.06)$ (Figure 5).

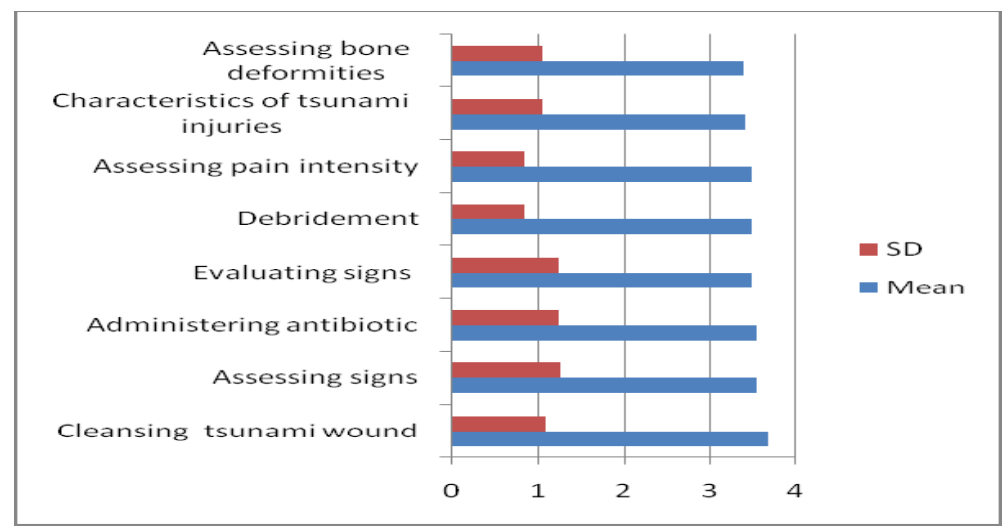

Figure 5. The Levels of Perceived Wound Care Skills 


\section{Discussion}

There were 97 nurses working in dr. Zainoel Abidin general hospital in Banda Aceh, Indonesia who participated in the study. The findings of the study are discussed as follow:

Overall, the findings of this study found that respondents' perceived clinical skills for tsunami care were at a moderate level. There are several reasons might have contributed to the findings of perceived clinical skills for tsunami care in this study.

Educational level of the respondents may have played an important role in the results. Firstly, the majority of the respondents $(78.4 \%)$ were educated at diploma level only which may have indicated a limited existing knowledge and skill for tsunami response, insufficiency in skill to seeking for appropriate and relevant information, and limited capability to develop further advanced clinical skills. The respondents who only have formal education at the diploma level might not have enough mastery on their knowledge to prepare themselves on the appropriate skills for tsunami care in the response phase.

A recent study found that the nurses whose education was at diploma level showed lower levels of knowledge and skill than baccalaureate, master or doctoral students in clinical management [25]. Moreover, the nurses educated at diploma level showed lower level in theoretical and clinical components for collected the evaluation data, written assignment skill testing, and contribution to conference, self-assessment, and other types of nursing program than baccalaureate and master levels of nurses [26]. Similarly, [27] reported that the nurses who have at the baccalaureate level has strong analytical and creative capacities and better skills in communication, assessment, cultural sensitivity, resourcefulness, the ability to apply knowledge, and scientific reasoning than the diploma level. In addition, the level of education of the nurses might have influenced the nurses' role in responding to an emergency disaster and the willingness to respond [28].

Secondly, the reason that may have influenced the moderate level of perceived clinical skills for tsunami care is age. Almost half of the nurses in this study were aged less than 30 years (42.3\%). It could be proposed older nurses have increased clinical skills capability as a result of accumulated experience and knowledge in clinical practice and that younger, less experienced nurses did not have yet enough accumulated clinical skill experiences particularly for tsunami care with complicated problems and serious illnesses. This could imply that the young adults may not have enough capability and competency to perform specific and advanced clinical skills as needed in caring for tsunami patients in acute response phase. However, it is known that young adults have good memory capacity and more opportunity to learn and seek for adequate nursing care in caring for the tsunami patients, so further education may improve the nursing care of tsunami-inflicted wounds. These may have influenced the finding of this study.

Consistently, the previous studies by Marsiske and Willis (as cited in [29]) found that age may have influenced on clinical performance which explicitly emphasized experience and accumulated knowledge structure in maintaining adult's cognition. Young adults have higher memory capacity, speed of processing information and lower inhibition than older 
adults. In addition, in [25] found that young adults (26-30 years) have lower knowledge and skill levels than middle adults (31-40 years) in clinical management systems.

Thirdly, the reason that may have influenced the moderate level of perceived clinical skills for tsunami care is working experience. The finding of this study showed that nearly half of the respondents had working experience as a nurse for less than 5 years (46.4\%). Moreover, 80.4 percent of them had direct experience in caring for tsunami patients in acute response phase for three months in average, because at that time they were recruited and has started working as a nurse in the acute response phase of the tsunami in acute care, emergency and critical care setting.

This indicates that most of the respondents had gained direct clinical skills by transforming experience to performing the nursing skills for tsunami patients. However, they had limitation in the duration of direct clinical experienced in caring for tsunami patients in the acute and emergency response phases. In addition, many experienced nurses with working experience more than 5 years had used advanced clinical skills related to care to the tsunami patients in acute response phase. These advanced skills involve tsunami wound care, and care of patients with tsunami lung or tsunami related aspiration pneumonia due to near drowning.

Similarly, a previous study supports that the experience can help nurses gain sudden insights, acquire new views about the benefits of former learning, absorb information from others, and learn from mistakes and repeated action in similar situations. Experience is a determinant factor in transforming nurses' knowledge to skill [30]. The findings of this study are in accordance with a recent study that showed nurses who had less than 5 years experience showed lower levels of knowledge and skill than those who had experience for more than 10 years nursing experience [25].

Furthermore, the finding of this study was consistent with a previous study by Watson (as cited in [31]) identified three criteria for experience: 1) the passage of time, 2) gaining skills or knowledge, and 3) exposure to an event. Passing of time is commonly used to define experiences in nursing where years of experience are used to categorize the skill of nurses. In addition, in [30] mentioned that the nurses who have worked for half year on resuscitation advanced life support (ALS) training were found to have increased knowledge and skills. In reference [32] also found that most of the nurses who have worked for many years and long periods of practices have high expectations of efficiency and mastery while performing the nursing practice.

Fourthly, the reason that could explain the finding on the moderate level of perceived clinical skills for tsunami care is training and education. Attending emergency training for basic life support (BLS), advanced cardiac life support (ACLS), basic trauma life support (BTLS), and disaster management are important to improve tsunami care skills of the nurses. The finding of this study showed that more than one-third $(37.1 \%)$ of the respondents had attended only once on the six different topics related to tsunami care of BLS, ACLS, BTLS, disaster management, infection control and prevention, and mental health care for tsunami survivors training. Moreover, 11.3 percent of the respondents were 
never trained. This could be interpreted that training on emergency response and disaster management for tsunami care were insufficient and lack of continuity).

Consistently, in [33] suggested that emergency training and education should be continued to enable the nurses to develop and apply knowledge and skills to meet demands of their current roles and functions needed for disaster response. Moreover, in [28] mentioned that the nurses need more training and preparation for appropriate response to emergency and disaster. Training and education sessions should address the barriers and perceptions that an event is unlikely to occur and could increase skills of nurses to respond to the disaster. In [34] suggested that emergency training of BLS and ACLS needs to improve by more frequency refresher trainings and allow more times for hand on skills practice. In reference [35] mentioned that the disaster and emergency training provide specific and unique skills set, yet will need to be performed efficiently to respond to emergency or disaster. Lastly, training for emergency medical service (EMS) can result in significant quantifiable team and individual skill gains for a range professional [36].

Lastly, the reason that might have contributed to the moderate level on the finding of the study is attending hospital disaster drill. Approxeimately half of the respondents $(42.3 \%)$ had attended hospital disaster drill provided by the hospital only once. This indicates that although the respondents have attended only once in their hospital drills, they have gained knowledge and skills to respond to disaster. Attending once on hospital disaster drill might not really influence the clinical skills for tsunami care of the nurses. Frequent and regular attendance on hospital disaster drills at least every one or two years could prepare the nurses with clinically rich knowledge and skills to respond to disaster events. Hospital drill is an essential part of hospital disaster preparedness to provide the sufficient nursing skills in response to disaster. The study in [37] figured out that hospital disaster drill was widely used throughout the world and considered a fundamental tool for evaluation and improvement of disaster response capacity for health care provider including nurses.

Several studies mentioned that disaster drill could provide sufficient knowledge and skills of nurses when responding to mass casualty incidents or disaster. Previous studies supported that disaster drill had equivalent results in prompting critical actions in mass casualty drills and increase the perceived reality to respond to disaster. To respond to mass casualty incidents or disaster, adequate training must be established, implemented, and maintained to ensure their safety and the optimal care of their patients [39]. Moreover, disaster drill also provide the basic tenets of disaster management and triage, decontamination, communication, incident command, and transport patients, and ability to rapidly form multidisciplinary work teams that communicate and function effectively to increase skills in disaster response [38].

Demographic data might have contributed to respondents' perceived clinical skills including diploma level, age, working experience as a nurse, and direct experience in caring for tsunami patients. These factors played an important role in order to improve respondents' perceived clinical skills in caring for tsunami patients. 


\section{Conclusion}

This study employed the descriptive-explorative study to describe the level of perceived clinical skills for tsunami care, among nurses in Banda Aceh, Indonesia. This study was conducted in a general hospital in Banda Aceh, Indonesia. Ninety-seven nurses who worked in hospital of the tsunami-affected area were recruited using the systematic random sampling. The respondents were required to complete a set of questionnaires, which consisted of three parts: 1) the Demographic Data Questionnaire [DDQ], 2) the Nurses' Clinical Experience Questionnaire [NCEQ], 3) the Tsunami Care Questionnaire [TCQ].

A pilot study was conducted and the desired alpha coefficient of .93 for the Nurses' Clinical Experience Questionnaire and .98 for the Tsunami Care Questionnaire were found. The data were analyzed by using descriptive statistics.

\section{Summary of the study findings}

The sample consisted of 97 nurses with the mean age of $31.9(\mathrm{SD}=6.6)$. Majority of them were more than 30 years old (56.7\%), and around three fourth of them were married $(75.3 \%)$. The majority of the respondents were female $(71.1 \%)$, were educated to diploma level $(78.4 \%)$, and all of them were Islam $(100 \%)$. Attending emergency training and education had six index score $(37.1 \%)$ with the mean score of $4.3(\mathrm{SD}=1.9)$. Working experience as a nurse of the respondents was more than 5 years $(53.6 \%)$ with the mean score of $8.7(\mathrm{SD}=$ 7.5). The majority of the respondents had experience in caring for tsunami patients $(80.4 \%)$.

The perceived clinical skills for tsunami care of the respondents showed that the highest mean score was acute respiratory care $(\mathrm{M}=3.57, \mathrm{SD}=0.86)$ with moderate levels and followed by wound care $(\mathrm{M}=3.50, \mathrm{SD}=0.96)$ with moderate levels as well.

\section{Limitation of the study}

The findings of this study cannot be generalized on the actual clinical skills for tsunami care, because this study was conducted only for perceived clinical skills for tsunami care among nurses who worked in the tsunami-affected area in a general hospital in Banda Aceh, Indonesia.

\section{Recommendations}

- Nursing Practice

The finding of this study recommends that the clinical practitioners and hospital policy makers should be aware of the importance on the preparation of clinical skills in response to the tsunami. The nurses' clinical skills for tsunami care needs improvement on knowledge and skills by attending trainings and educational programs and hospital disaster drills regularly. Furthermore, clinical practitioners should encourage nurses to increase their skills in caring for tsunami patients, 
attending emergency training and education, and attending hospital disaster drill regularly to meet a standard level on preparations in response to the disaster that can happen basically anywhere in the world and particularly in the island countries that highly risk for tsunami disaster.

- Nursing Education

The findings of this study could serve as evidence or guideline for nurse educators to better prepare nursing students about for nurses' knowledge and skills for tsunami care by providing disaster and emergency trainings. Those training consist of disaster management, basic life support (BLS), advanced cardiac life supports (ACLS), advanced trauma life supports (ATLS), psychological care for tsunami victims, and infection control and prevention in response to tsunami disaster in the hospital setting. Also, the nurse educators can well prepare for the students for exploration the local knowledge and skills of the community that can help for mitigation and response of the disaster in the future by cultural, social, geographical and geological approaches.

- Nursing Research

These findings present valuable information about perceived clinical skills for tsunami care and its related factors among nurses. Particularly, the emergency training and research center should consider developing and continuing the training program and hospital disaster drill. The hospital and emergency training and research center should also allocate budget for hospital disaster plan to improve nurses' skills in response to disaster. Further study is needed to develop practical guidelines of clinical skills for tsunami care in acute response phase in the hospital setting.

\section{Author details}

\section{Cut Husna}

Medical \& Surgical Nursing Department, Nursing School, Faculty of Medicine, Syiah Kuala University, Darussalam-Banda Aceh, Indonesia

\section{Acknowledgement}

Thanks to all of the nurses' who worked in Dr. Zainoel Abidin general hospital Banda Aceh, Indonesia for their participation. Also gratefully thanks to the Director of the Institute for Research and Development on Health and Epidemiology Unit, Faculty of Medicine, Prince of Songkla University, and Graduate School, Prince of Songkla University, Thailand for their assistance for partial granting support to this study.

\section{References}

[1] World Health Organization (2005). The emergency and its aftermath in moving beyond the tsunami. New Delhi- India: World Health Organization Office for South-East Asia. 
[2] Garfield, R., \& Hamid, A. Y. (2006). Tsunami response: A year later. American Journal of Nursing, 106(1): 76-79.

[3] Maegele, M., Gregor, S., Yuecel, N., Simanski, C., Paffrath, T., Rixen, D., et al. (2006). One year ago not business as usual: Wound management, infection and psychoemotional control during tertiary medical care following the the 2004 Indian Ocean Tsunami disaster in southeast Asia. Critical Care 1(2): 1-9.

[4] Collander, B., Green, B., Millo, Y., Shamloo, C., Donnellan, J., \& DeAtley, C. (2007). Development of an "All-Hazards" hospital disaster preparedness training course utilizing multi-modality teaching. Prehospital and Disaster Medicine, 63-68.

[5] Veenema, T. G. (2006). Expanding educational opportunities in disaster response and emergency preparedness for nurses. Nursing Education Perspectives, 27(2): 93-99.

[6] Chaya, M. (2005). Relieving suffering after the tsunami. Nursing, 35(8): 48-49.

[7] Stotts, N. A., Puntillo, K., Morris, A. B., Stanik-Hutt, J., Thompson, C., White, C., et al. (2004). Wound care pain in hospitalized adult patients. Heart and Lung, 33(5).

[8] Lukthitikul, S., \& Hatthakit, U. (2007). Wound care for tsunami victims of nurses at hospitals of Phang-Nga province. Songklanagarind Medical Journal, 25(5): 391- 400.

[9] Rebmann, T., Carrico, R., \& English, J. F. (2008). Lessons public health professionals learned from past disasters. Public Health Nursing, 25(4): 344-352.

[10] International Nursing Coalition for Mass Casualty Education (2003). Educational competencies for registered nurses responding to mass casualty incidents. New York: International Nursing Coalition for Mass Casualty Education.

[11] Kaewlai, R., Meennuch, W., Srisuwan, T., Prasitvoranant, W., Yenarkarn, P., \& Chuapetcharasopon, C. (2009). Imaging in tsunami trauma. Journal of Medical Ultrasound, 17(1): 1-8.

[12] Prasartritha, T., Tungsiripat, R., \& Warachit, P. (2008). The revisit of the 2004 Indian Ocean Tsunami in Thailand: Characteristics of wounds. International Wound Journal 5(1): 8-19.

[13] Wacharong, C., Chukpaiwong, B., \& Mahaisavariya, B. (2005). After the tsunamis. The Lancet, 365(9455), 203-203.

[14] Potera, C. (2005). In disaster's wake: Tsunami lung. Environmental Health Perspectives, 113(11): A734-A734.

[15] World Health Organization (2005). Epidemic-prone disease surveillance and response after the tsunami in Aceh Province, Indonesia. Weekly Epidemiological Record, 80(18): 160-164.

[16] Harcombe, C. (2004). Nursing patients with ARDS in the prone position. Nursing Standard, 18(19): 33-39.

[17] Kozier, B., Erb, G., Berman, A., \& Snyder, S. (Eds.). (2004). Fundamentals of nursing: Concepts, process, and practice (7 th ed.). New Jersey.

[18] Geertruid, M. H., Marres, M., W., D., de Lange, M., P.H., L., Leenen, M., et al. (2006). Wound infections in repatriated survivors of the tsunami disaster. 18(4): 1-5.

[19] Ignatavius, D. D., \& Workman, M. L. (2006). Medical surgical nursing critical thinking for collaborative care (5 th ed.). St. Loius. Missouri: Elsevier Saunders.

[20] Scott, L. (2009). Fracture management. Nursing Standard, 23(27): 59-59 
[21] Johnson (2005). Measuring pain Visual Analog Scale versus Numeric Pain Scale: What is the difference? Journal of Chiropractic Medicine, 4(1): 43-44.

[22] Dunwoody, C. J., Krenzischek, D. A., Pasero, C., Rathmell, J. P., \& Polomano, R. C. (2008). Assessment, physiological monitoring, and consequences of inadequately treated acute pain. Journal of PeriAnesthesia Nursing, 23(1, Supplement 1), S15-S27.

[23] Sloan, K., \& Summers, A. (2006). Tetanus vaccination: The issue of 'just in case' vaccinations in emergency departments. Australasian Emergency Nursing Journal, 9(1): 35-38.

[24] Polit, D. F., \& Hungler, B. P. (1999). Nursing research: Principles and methods (6 th ed.). Philadelphia: Lippincott.

[25] Chan, M. F. (2009). Factors affecting knowledge, attitudes, and skills levels for nursing staff toward the clinical management system in Hong Kong. Computers Informatics Nursing 27(1): 57-65.

[26] Oermann, M., Yarbrough, S., Saewert, K., Ard, N., \& Charasika, M. (2009). Clinical evaluation and grading practices in schools of nursing: National survey findings part II. Nursing Education Perspectives, 30(6): 352.

[27] Alonzo, A. (2009). Motivational factors in registered nurses completing a baccalaureate completion program. Unpublished Ph.D., University of Kansas, United States, Kansas.

[28] Evers, S., \& Puzniak, L. (2005). Bioterrorism knowledge and emergency preparedness among school nurses. The Journal of School Health, 75(6): 232.

[29] Kliegel, M., \& Martin, M. (2007). Adult age differences in errant planning: The role task familiarity and cognitive resources. Experimental Aging Research 33: 145-161.

[30] Jensen, M. L., Lippert, F., Hesselfeldt, R., Rasmussen, M. B., Mogensen, S. S., Jensen, M. K., et al. (2008). The significance of clinical experience on learning outcome from resuscitation training: A randomized controlled study. Resuscitation, 80: 238-243.

[31] Considine, J., Botti, M., \& Thomas, S. (2007). Do knowledge and experience have specific roles in triage decision-making. Academic emergency Medicine, 14: 722-726.

[32] Bjork, I. T., \& Kirkevold, M. (1999). Issues in nurses' practical skill development in the clinical setting. Journal of Nursing Care Quality, 14(1): 72-84.

[33] Gould, D., Berridge, E.-J., \& Kelly, D. (2007). The national health service knowledge and skills framework and its implications for continuing professional development in nursing. Nurse Education Today, 27(1): 26-34.

[34] Smith, K. K., Gilcreast, D., \& Pierce, K. (2008). Evaluation of staff's retention of ACLS and BLS skills. Resuscitation, 78(1): 59-65.

[35] Kobayashi, L., Shapiro, M. J., Suner, S., \& Williams, K. A. (2003). Disaster medicine: The potential role of high fidelity medical simulation for mass casualty incident training. Medicine and Health Rhode Island, 86(7): 196.

[36] Scott, J. A., Miller, G. T., Barry Issenberg, S., Brotons, A. A., Gordon, D. L., Gordon, M. S., et al. (2006). Skill improvement during emergency response to terrorism training. Prehospital Emergency Care, 10: 507-514.

[37] Green, G. B., Modi, S., Lunney, K., \& Thomas, T. L. (2003). Generic evaluation methods for disaster drills in developing countries. Annals of Emergency Medicine, 41(5): 689699. 
[38] Kobayashi, L., Shapiro, M. J., Suner, S., \& Williams, K. A. (2003). Disaster medicine: The potential role of high fidelity medical simulation for mass casualty incident training. Medicine and Health Rhode Island, 86(7): 196.

39] Gillett, B., Peckler, B., Sinert, R., Onkst, C., Nabors, S., Issley, S., et al. (2008). Simulation in a disaster drill: Comparison of high-fidelity simulators versus trained actors. Academic Emergency Medicine, 15: 1144-1151.

[40] Dirkes, S. (2002). Help for ARDS patients. RN, 65(8): 52-58.

[41] Baranoski, S. (2008). Choosing a wound dressing, part 1. Nursing, 38(1), 60-61.

[42] Mendez-Eastman, S. (2005). Wound dressing categories. Plastic Surgical Nursing Retrieved 2, 25, from http://www.dressings.org/Dressings/telfa.htm

[43] Black, J. M. (2005). Medical surgical nursing clinical management for outcomes $\left(7^{\text {th }}\right.$ ed. Vol. 1). St. Louis: Elsevier Senders.

[44] Stevensen, C. (1995). Nonpharmacological aspects of acute pain management. Complementary Therapies in Nursing and Midwifery, 1(3): 77-84.

[45] Morrison, G. (2007). Understanding shock. 3(3), 121-125. 